\title{
Solid State Cultivation of Hericium erinaceus Biomass and Erinacine: A Production
}

Blaz Gerbec ${ }^{1}$, Eva Tavčar², Andrej Gregori ${ }^{3}$, Samo Kreft ${ }^{2}$ and Marin Berovic ${ }^{*}$

${ }^{1}$ Department of Chemical, Biochemical and Environmental Engineering, Faculty of Chemistry and Chemical Technology, University of Ljubljana, Askerceva 5, SI-1115 Ljubljana, Slovenia

${ }^{2}$ Department of Pharmaceutical Biotechnology, Faculty of Pharmacy, University of Ljubljana, Aškerčeva 7, SI-1115 Ljubljana, Slovenia

${ }^{3}$ Institute for Natural Science (Zavod za naravoslovje), Ulica Bratov Ucakar 108, SI-1000 Ljubljana; MycoMedica d.o.o. Podkoren 72, SI-4280 Kranjska Gora, Slovenia

\begin{abstract}
Hericium erinaceus is a medicinal mushroom producing biologically active metabolite erinacin $\mathrm{A}$. In this research we compared fungal growth and metabolite production in different reactor types and different substrates with added casein peptone and $\mathrm{NaCl}$. Cultivation of fungal biomass was performed in glass jars and horizontal stirred tank reactor. The cultivation in reactor was carried out at $24^{\circ} \mathrm{C}$ and airflow $5 \mathrm{~L} / \mathrm{min}$. Periodical mixing of $\mathrm{N}=80 \mathrm{rpm}$, was used. Solid state substrate based on mixture of husked millet and paddy millet was used. For simultaneous determination of erinacine $A$ and ergosterol contents in a single sample new analytical method was developed. The amount of biomass obtained in glass jars averaged at $100 \mathrm{mg} / \mathrm{g} \pm 12 \mathrm{mg} / \mathrm{g}$, while in horizontal mixing bioreactor it easily raised up to $350 \mathrm{mg} / \mathrm{g}$. The highest erinacin A concentration was detected in substrate with $0.56 \% \mathrm{NaCl}$ and $3.4 \%$ casein peptone content.
\end{abstract}

Keywords: Solid state cultivation; Horizontal stirred tank reactor; Hericium erinaceus; LC-MS; Erinacine A; Ergosterol

\section{Introduction}

Medicinal properties of Hericium erinaceus (Bull.: Fr) Pers. (also known as lion's mane, monkey's head, hedgehog fungus, pom pom blanc and yamabushitake) have been well-known for hundreds of years in traditional Chinese and Japanese cuisine as well as herbal medicine to treat various human diseases [1]. Fungi of the genus Hericium contain various ingredients with antibacterial activity, cytotoxic effect on cancer cells and compounds that stimulate the synthesis of the Nerve Growth Factor (NGF). Although quite a few species of Hericium are known, the fruiting bodies cultivation on the waste sawdust was developed for H. abietis and H. erinaceus only [2].

In $H$. erinaceus various pharmaceutically active substances were found such as phytosterols ( $\beta$-sitosterol and ergosterol), which lower the content of Low-Density Lipoproteins (LDL) and triglycerides that operate anticarcinogenic and as well reduce the metabolism of fats [3]. The fruit body is composed of numerous constituents such as polysaccharides, proteins, lectins, phenols, hericenones, erinacines and terpenoids. They strengthen the immune system, relieve gastritis and gastrointestinal infections, reflux and upset stomach due to stress [1,4].

$H$. erinaceus water-soluble polysaccharides increase activity of macrophages and other immune cells in the fight against cancer cells, but also demonstrate reduction of metastases formation. The most outstanding activity of $H$. erinaceus extract is that it strengthens the immune system and activates NGF synthesis [5]. Due to the increased proliferation of $\mathrm{T}$ and B-lymphocytes it strengthens the immune system and strengthens the body's natural defenses, thus expressing very positive effects on cancer patient's life quality [3].

Among the compounds isolated from $H$. erinaceum fruiting bodies and cultured mycelia, most interesting are the low-molecular-weight compounds belonging to a group of cyathin diterpenoids (erinacines A-K, P, and Q). Several of them, i.e. erinacines A-H, are known to have a potent stimulating effect on Nerve Growth Factor (NGF) synthesis in vitro [6-12].

NGF is an essential protein supporting growth and maintenance of peripheral sympathetic neurons. Due to degeneration of neurons NGF synthesis stimulators can be applied as potential drugs against central nervous system disorders, such as Alzheimer's disease [1].

Erinacine A, isolated from the cultured mycelia of $H$. erinaceum, the main representative of this compounds group, has a strong enhancing effect on NGF synthesis, much stronger than epinephrine [6]. Furthermore, this compound increases catecholamine and NGF content in the central nervous system of rats [13-15].

Erinacines and hericenons reduce anxiety as well as depression. For an accurate understanding of the overall mechanism of $H$. erinaceus diterpenoids action it requires additional clinical studies with physiological markers, such as hormones, or more profounded studies of autonomic nervous activity [16].

The structure of erinacine A was determined for the first time through the observation of chemical reactions, NMR and IR spectroscopy, and mass spectrometry data [6]. Krzyczkowski et al. [18] developed an isocratic HPLC method for quantitative determination of erinacine A [17]. Erinacine A was described in the form of white crystals [6] and yellow amorphous powder with the melting point of $72-74^{\circ} \mathrm{C}[18,19]$.

Besides reports on $H$. erinaceus fruiting bodies cultivation, reports on biomass cultivation in bioreactors, particularly in solid state reactors, are rare. In present research, cultivation of $H$. erinaceus was optimized in glass jars and transferred to Horizontal Stirred Tank Reactor (HSTR). Fast gradient HPLC analytical method for simultaneous determination of erinacine A and ergosterol was determined.

${ }^{*}$ Corresponding author: Marin Berovic, Department of Chemical, Biochemical and Environmental Engineering, Faculty of Chemistry and Chemical Technology, University of Ljubljana, Askerceva 5, SI-1115 Ljubljana, Slovenia, Tel: +386-12419-510; E-mail: marin.berovic@fkkt.uni-lj.si

Received January 26, 2015; Accepted February 20, 2015; Published February 24, 2015

Citation: Gerbec B, Tavčar E, Gregori A, Kreft S, Berovic M (2015) Solid State Cultivation of Hericium erinaceus Biomass and Erinacine: A Production. J Bioprocess Biotech 5: 210 doi: 10.4172/2155-9821.1000210

Copyright: $\odot 2015$ Gerbec B, et al. This is an open-access article distributed under the terms of the Creative Commons Attribution License, which permits unrestricted use, distribution, and reproduction in any medium, provided the original author and source are credited. 


\section{Materials and Methods}

\section{Microorganism}

Hericium erinaceus strain He Erin (Institute for Natural Sciences, Slovenia) was used in all of the experiments. Strain was maintained on Potato Dextrose Agar (Biolife, Italy) at $24^{\circ} \mathrm{C}$. It was maintained active by regular (every 14 day) transfers on fresh Potato Dextrose Agar plates.

\section{Cultivation in glass jars}

Substrate was based on mixture of husked and paddy millet in ratio=7: 2 . The impact of $\mathrm{NaCl}$ and casein peptone, as basic additives of the medium was examined. Additives of $0,0.1,0.2$ and $0.5 \mathrm{~g}$ of $\mathrm{NaCl}$ and $0,1,3$ and $7 \mathrm{~g}$ of casein peptone to $88.8 \mathrm{~g}$ of dry media were used. The addition of $\mathrm{NaCl}$ to media corresponds to $0,0.11,0.23$ and $0.56 \%$ and casein peptone addition corresponds 1.1, 3.4 and $7.9 \%$ of dry media weight respectively. The total amount of the substrate was in all cases $180 \mathrm{~g}$, with $50 \%$ water content. Substrate were filled into jars and sterilized for 1 hour at $\mathrm{T}=121^{\circ} \mathrm{C}$ and pressure of $\mathrm{P}=1.2 \times 10^{5} \mathrm{~Pa}$.

Cultivation proceeded for 5,6 and 7 weeks at $24^{\circ} \mathrm{C}$. All of the experiments were managed in triplicates. In total, 144 jars were used ( 4 different $\mathrm{NaCl}$ contents $\times 4$ different casein peptone content $\times 3$ cultivation times $\times 3$ replicates). After cultivation the glass content was dried for $100 \mathrm{~h}$ at $60^{\circ} \mathrm{C}$ to achieve constant moisture content.

\section{Cultivation in horizontal stirred tank reactor}

Experiments were carried out in a 15 L Horizontal Stirred Tank Reactor (HSTR) of our own construction and design [20]. The cultivation was carried out at $24^{\circ} \mathrm{C}$ and $5 \mathrm{~L} / \mathrm{min}$ airflow. Periodical mixing of $\mathrm{N}=80 \mathrm{rpm}$, for 2 minutes (in the first 7 days every second day, and every day in the last part of cultivation) was used. A moistening air device with sterile distilled water was connected to the bioreactor to prevent the drying of the substrate (Figure 1).

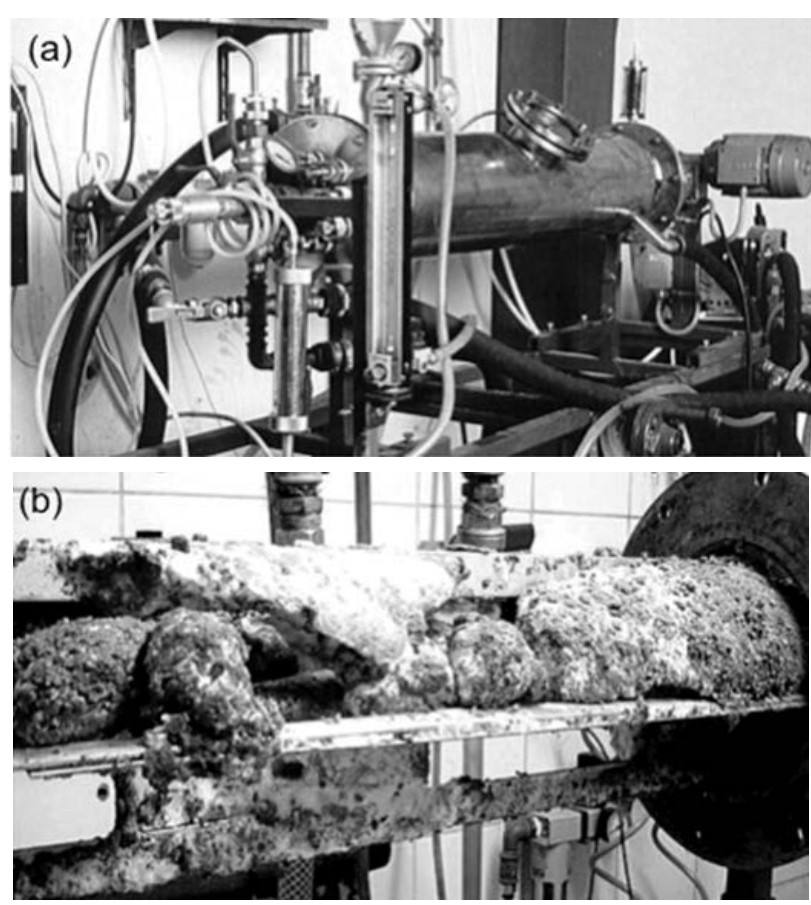

Figure 1: (a) Horizontal Stirred Tank Reactor, (b) The growth of fungal biomass in horizontal stirred tank reactor after 8 week of cultivation.
The cultivation substrate consisted of beech sawdust, paddy millet and hulled millet. The ratio paddy millet: husked millet in the substrate was $1: 1$ and substrate water content $50 \%$. In solid state reactor in contrary with glass jars aeration of the solid matrix was applied. Sawdust (10\%) was added to the substrate to increase the porosity and oxygen permeability. In accordance with the results of substrate optimization in the glass jar experiments, the addition of $\mathrm{NaCl}$ was $0.56 \%$ and casein peptone $3.4 \%$ (dry weight/dry weight).

Inoculum for the cultivation was prepared from a mixture of beech sawdust and paddy millet in the ratio 4: 1 incubated and overgrown with $H$. erinaceus mycelia. It represented $7 \%$ of the total substrate weight. Cultivation of fungal biomass in HSTR proceeded for 8 weeks.

\section{Analytical methods}

Biomass: For determination of biomass on solid particles, ergosterol assay was applied in all the experiments [21]. Ergosterol in fungi is included as an integral part of the cell membrane and therefore a relevant indicator of the fungal biomass content in solid matrix [22]. For determination of ergosterol, HPLC method was developed and described in Chromatographic analysis at the Results and discussion section.

Electron microscopy: For monitoring of microbial growth, Field Emission Scanning Microscopy (FE-SEM, Supra 35, Carl Zeiss, Germany) equipped with energy dispersive spectroscopy Oxford INCA 400, UK was used.

\section{Extraction of ergosterol and erinacin A}

$2 \mathrm{~g}$ of dry overgrown substrate sample was milled and extracted with $20 \mathrm{ml}$ of solvent. $30 \%$ methanol and ethanol were tested as solvents for the extraction of erinacine A and ergosterol. Ethanol proved as a better solvent and was further tested in concentrations of $20,50,70$, 85 and $96 \%$. $70 \%$ concentration was confirmed to be optimal. The extracts were sampled after 1,4 and $24 \mathrm{~h}$ of the extraction and then reextracted with fresh solvent for 2 and $120 \mathrm{~h}$. The extraction conditions were further optimized in a test, comparing three procedures: 1) the maceration on a shaker (150 rpm, room temperature), 2) maceration on a shaker ( $150 \mathrm{rpm}$, room temperature) with additional $10 \mathrm{~min}$ sonification, and 3) maceration in a water bath $\left(50^{\circ} \mathrm{C}\right)$ with three additional $10 \mathrm{~min}$ shakings ( $10 \mathrm{~min}, 150 \mathrm{rpm}$ on a shaker). The $24 \mathrm{~h}$ extraction in a water bath $\left(50^{\circ} \mathrm{C}\right)$ was selected as an optimal procedure. Prior to HPLC analysis, all samples were centrifuged ( $3 \mathrm{~min}, 4192.5 \mathrm{~g}$ ) and filtered through a $0.22 \mu \mathrm{m}$ filters (Supelco, PTFE).

\section{Chromatographic analysis}

The HPLC system (Shimadzu Prominence, Kyoto, Japan) consisted of a system controller (CBM-20A), column oven (CPO-20AC) and a solvent delivery pump with a degasser (DGU-20A5) connected to a refrigerated auto sampler (SIL-20AC) with a Photo Diode Array detector (SPD-M20A) that monitored the wavelengths $190-800 \mathrm{~nm}$. The responses of the detectors were recorded using LC Solution software version $1.24 \mathrm{SP} 1$. The chromatography was performed at $40^{\circ} \mathrm{C}$ and a flow rate of $1.5 \mathrm{~mL} / \mathrm{min}$ using a Phenomenex Kinetex $\mathrm{C} 18$ column $(10 \mathrm{~cm} \times 4.6 \mathrm{~mm}$ I.D., $2.7 \mu \mathrm{m}$ particle size $)$. The following gradient method using water (solvent A) and acetonitrile (solvent B), both containing $0.1 \%$ formic acid, was utilized: $0.01-3.00$ (30-50\% B), 3.009.00 (50-60\% B), 9.01-18.00 (60-100\% B), 18.01-23.00 (30\% B). Since the standard od erinacine A was not available to make a calibration curve, all quantitative results on erinacin A content are expressed as the area under the curve (AUC) of erinacin A chromatogram peak. 


\section{Mass spectrometric analysis}

The chromatographic separation was performed on a Waters Acquity ultra-performance liquid chromatograph ${ }^{\odot}$ (Waters Corp., Milford, MA, USA) with a column identical to that used for the quantitative HPLC analysis. The flow rate was adjusted according to the capacity of the mass detector to $0.5 \mathrm{~mL} \mathrm{~min}$. The following gradient method was utilized with MilliQ water containing $0.1 \%$ formic acid (solvent A) and acetonitrile (solvent B): 0.01-9.00 (3050\% B), 9.00-27.00 (50-60\% B), 27.01-54.00 (60-100\% B), 54.01-69.00 $(30 \% \mathrm{~B})$. The injection volume was $10 \mu \mathrm{L}$, and the column temperature was maintained at $40^{\circ} \mathrm{C}$. The LC system was interfaced with a hybrid quadrupole orthogonal acceleration time-of-flight mass spectrometer (Q-ToF Premier, Waters, Milford, MA, USA). The compounds were analyzed under positive (ESI (+)) and negative (ESI(-)) ion conditions. The capillary voltage was set at $3.0 \mathrm{kV}$, while the sampling cone voltage was $20 \mathrm{~V}$. The source and desolvation temperatures were 120 and $200^{\circ} \mathrm{C}$, respectively. The nitrogen desolvation gas flow rate was $500 \mathrm{~L}$ $\mathrm{h}^{-1}$. The acquisition range was between $\mathrm{m} / \mathrm{z} 50$ and 1000 with argon serving as the collision gas at a pressure of $4.5 \cdot 10^{-3} \mathrm{mbar}$ in the T-wave collision cell. The data were collected in centroid mode, with a scan accumulation time of $0.2 \mathrm{~s}$ and an interscan delay of $0.025 \mathrm{~s}$. The data station utilized the Mass Lynx v4.1 operating software. Accurate mass measurements were obtained with an electrospray dual sprayer using the reference compound leucine enkephalin $\left([\mathrm{M}+\mathrm{H}]^{+}=556.2271\right)$ at a high mass resolution of 10,000.

\section{Statistics}

All of the cultivation experiments were performed at least in three runs and the average values were calculated from the sum of the data. Data were expressed as mean \pm S.D. The results were analyzed for statistical significance by one-way analysis of variance (ANOVA) test using the Statistical Package of the Social Science (SPSS) version 11.0 (SPSS Inc., Chicago, IL, USA). Statistical significance of the test effects was evaluated at $\mathrm{p}<0.05$.

\section{Results and Discussion}

\section{Growth optimization in glass jars}

First step were substrate optimization experiments. In glass jar cultivation, the influence of $\mathrm{NaCl}$ and casein peptone addition was studied. Mycelial growth in the glass jars was very intensive. After extended adaptive phase of 2 weeks, first mycelium appears, at 5 weeks mycelia overgrew half of the substrate height and after 8 weeks the whole substrate volume was overgrown.

The best production of erinacine A $225,4 \times 10^{3}$ AUC was indicated in 8 weeks of cultivation in the substrate containing a combination of $0.56 \% \mathrm{NaCl}$ and $3.4 \%$ casein peptone (Figure 2). In the control substrate without additions, the biomass growth as well as the biosynthesis of erinacine A completely stopped after 2 weeks of cultivation. The amount of obtained biomass in glass jars was in average about 100 $\mathrm{mg} / \mathrm{g} \pm 12 \mathrm{mg} / \mathrm{g}$.

\section{Cultivation in horizontal stirred tank reactor}

In first HSTR experiments the same substrate mixture composition optimized already in glass jar experiments were used. In HSTR a problem of water condensate on the bottom of reactor tube occurred. Therefore $10 \%$ of beech sawdust was added to paddy millet and hulled millet substrate to absorb the water condensate. Sawdust was added to the substrate also to increase solid bed porosity and oxygen

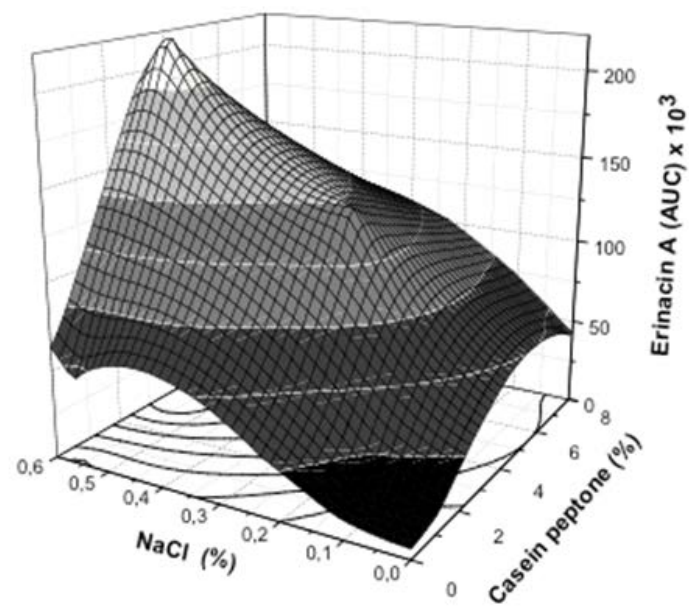

Figure 2: The impact of $\mathrm{NaCl}$ and casein peptone on erinacine $\mathrm{A}$ content in 8 week cultivation in glass jar

permeability. Permanent mixing could eliminate this problem, but it would disable the filamentous growth and biomass cluster formation. In contrary with glass jars cultivation the growth of fungal mycelia in HSTR proceeded at different condition. In solid state reactor aeration of solid matrix was applied during whole course of cultivation. Cultivation in HSTR proceeded for 8 weeks. At the end of cultivation substrate was completely overgrown in thick woody form (Figure 1b).

The SEM electron microscopy images had shown the growth situation and the porosity of overgrown solid matrix in HSTR Although mixing was periodically applied 2 minutes per day in the first 2 weeks of cultivation, SEM images (Figures 3a and 3b) shown that it damaged the filaments and inhibited the growth. At 5 weeks the substrate was already fixed to the impeller, so further disintegration did not occur. After 8 weeks of cultivation solid matrix was completely overgrown by mycelia (Figures $3 \mathrm{c}$ and $3 \mathrm{~d}$ ).

Although out the experiments in glass jar cultivation the best results of erinacine A production were obtained at the combination of $0.56 \%$ $\mathrm{NaCl}$ and 3.4\% casein peptone in HSTR, the highest concentration of ergosterol was obtained at 8 weeks of cultivation with addition of $0.56 \%$ $\mathrm{NaCl}$ and 2\% casein peptone. Compared with glass jar cultivation in HSTR cultivation higher yield of ergosterol content was obtained while no erinacine A was detected (Figure 4).

\section{Chromatographic analysis}

For simultaneous determination of erinacine A and ergosterol contents in a single sample, new method was developed. Erinacine A was eluted at $6.7 \mathrm{~min}$ and ergosterol at $15 \mathrm{~min}$ on the HPLC chromatogram (Figure 5a). The chromatograms were observed at 340 $\mathrm{nm}$ for erinacine A and a $281 \mathrm{~nm}$ for ergosterol. Erinacine A can also be detected at $281 \mathrm{~nm}$ when only one wavelength detection is possible, but this yields a lower sensitivity.

\section{Identification of the compounds}

The absorption spectrum of erinacine A (Figure 5b) exhibited maxima at 345 and $295 \mathrm{~nm}$ and a minimum at $253 \mathrm{~nm}$. A corresponding absorption maximum at $340 \mathrm{~nm}$ was previously reported [17]. Ergosterol was used for fungal biomass measurement. It was identified with the use of a standard compound and a characteristic absorption 


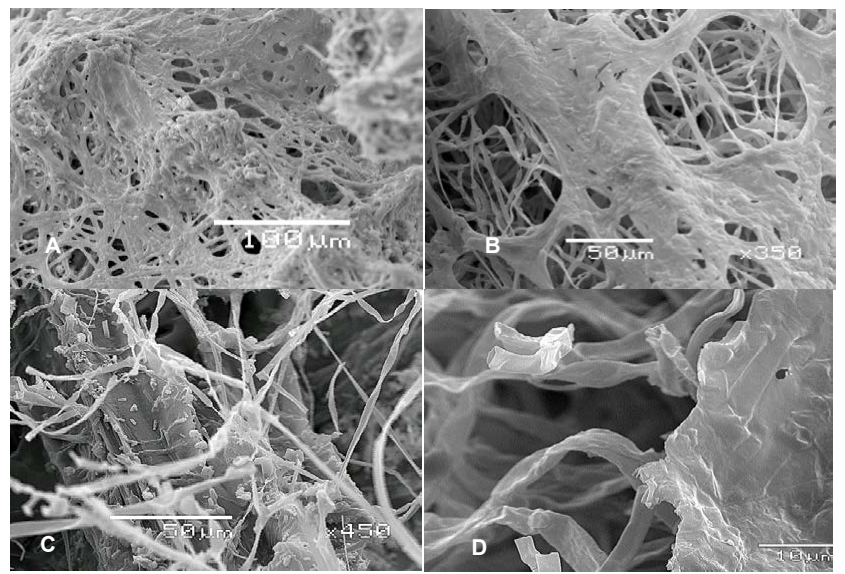

Figure 3: Hericium erinaceus growth mycelia in horizontal stirred tank reactor SEM electron microscope images of mycelial growth. A and B at 6 weeks, C at 7 weeks and $D$ at 8 weeks - thick mycelia cover at the end of cultivation.

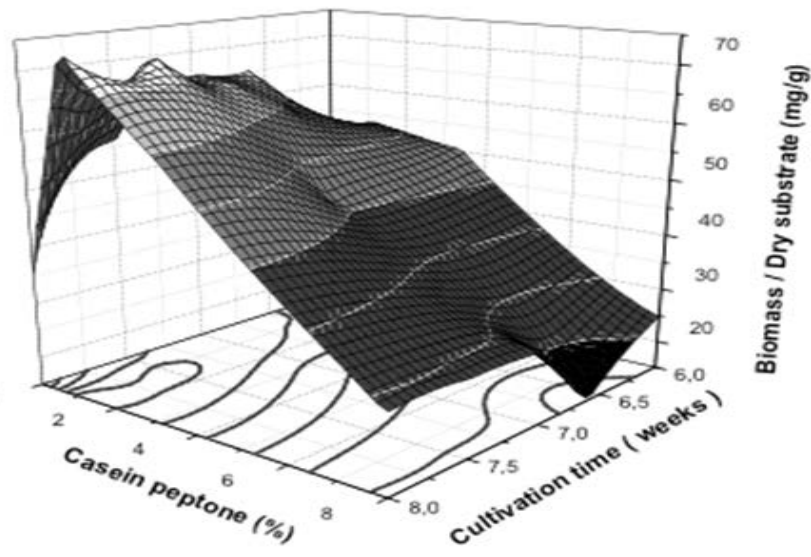

Figure 4: Production of biomass in HSTR on substrate with various addition of casein peptone and $0.56 \% \mathrm{NaCl}$

(a)

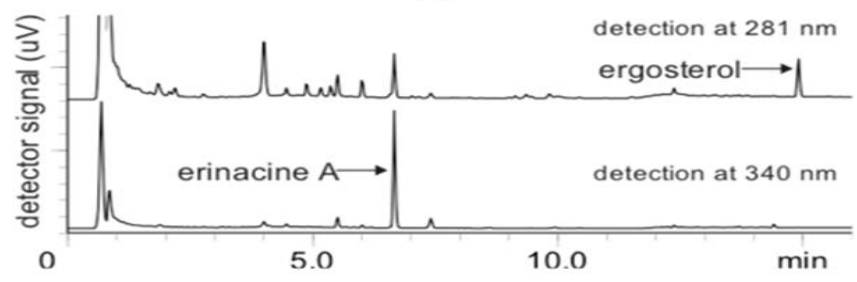

(b)

(c)

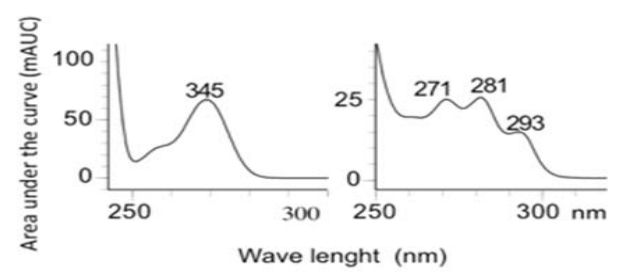

Figure 5: (a) HPLC chromatograms of a typical Hericium erinaceum extract including detection of ergosterol and erinacine A, (b) absorption of erinacine A and (c) ergosterol spectra with three characteristic maxima
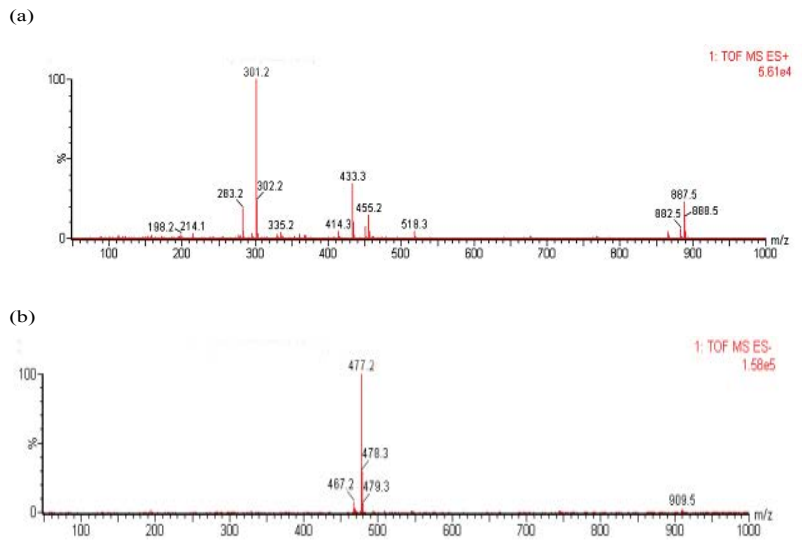

Figure 6: (a) LC-MS spectrum of erinacine A in TOF ESI+ mode (top), (b) ESI- mode (bottom), respectively.

spectrum with three maxima at wavelengths 271,281 and $293 \mathrm{~nm}$ and minima at 276 and $290 \mathrm{~nm}$ (Figure 5c).

The LC/MS measurements provided mass spectra with the basic information of molecular masses $\left([\mathrm{M}+\mathrm{H}]^{+}\right.$or $[\mathrm{M}-\mathrm{H}]^{-}$for the chromatographically separated compounds. The mass measurements were simultaneously obtained using a Q-TOF high resolution mass analyzer. The proposed elemental compositions of the ions were obtained from the accurate mass measurement data. A molecular ion $[\mathrm{M}+\mathrm{H}]^{+}$at $\mathrm{m} / \mathrm{z} 433$ with predicted elemental composition of $\mathrm{C} 25 \mathrm{H} 36 \mathrm{O} 6$ could be seen on the positive spectrum. Signals with $\mathrm{m} / \mathrm{z} 455$ and 887 represented molecular ion adducts, $[\mathrm{M}+\mathrm{Na}]^{+}$and $[2 \mathrm{M}+\mathrm{H}]^{+}$. An acetic acid adduct $[\mathrm{M}+\mathrm{HCOOH}]^{-}$formation with $\mathrm{m} / \mathrm{z} 477$ was observed on the negative spectrum. Our findings correspond and complement the literature data [6,17]. Using LC-MS, Erinacine A was detected at 340 $\mathrm{nm}$ at 6.7 th min (bottom) and ergosterol was detected at $281 \mathrm{~nm}$ at 15th min (top) (Figures 6a and 6b).

\section{Conclusions}

The extraction procedure and HPLC analytical method for simultaneous determination of erinacine $\mathrm{A}$ and ergosterol from Hericium erinaceum have been developed, enabling simultaneous quantification of active metabolite (erinacine A) content and determination of biomass content in the medium. These analytical methods are a very useful tool in determination of optimal growth conditions for $H$. erinaceus erinacine A and fungal biomass production on solid-state substrates.

Although the experiments in glass jars enable large number of experiments in the small-scale, they can be used as a compass for $H$. erinaceus fruiting bodies cultivation. Great advantage of growing in solid-state bioreactor is aeration, temperature and mixing control, representing a more suitable method for large-scale fungal biomass production. In present case, the amount of biomass obtained in glass jars was $100 \mathrm{mg} / \mathrm{g} \pm 12 \mathrm{mg} / \mathrm{g}$ on average, while in HSTR on sawdust containing substrates it easily raised up to $350 \mathrm{mg} / \mathrm{g}$.

In the glass jars up to $225,4 \times 10^{3} \mathrm{AUC}$ of erinacine A was produced, while in a HSTR on sawdust containing substrates there was no erinacine A production detected. The main reason for this could be the lack of precursors needed for erinacine A biosynthesis. Substrate used in HSTR also did not include enough starch to support the fungal metabolism in sense of erinacine A synthesis. In contrary with glass jars 
Citation: Gerbec B, Tavčar E, Gregori A, Kreft S, Berovic M (2015) Solid State Cultivation of Hericium erinaceus Biomass and Erinacine: A Production. J Bioprocess Biotech 5: 210 doi: 10.4172/2155-9821.1000210

in HSTR cultivation carbon dioxide was ventilated out of solid matrix and therefore eliminating its stimulatory effect and affecting erinacine A biosynthesis as well.

\section{References}

1. Mizuno T (1999) Bioactive Substances in Hericium erinaceus (Bull.:Fr.) Pers (Yamabushitake), and its Medicinal Utilization. Int J Med Mushrooms 1: 105119.

2. Stamets $P(2006)$ Can mushrooms help save the world? Interview by Bonnie J. Horrigan. Explore (NY) 2: 152-161.

3. Mizuno T (1995) Bioactive biomolecules of mushrooms: food function and medicinal effect of mushroom fungi. Food Reviews International 11: 7-21.

4. Wong KH, Sabaratnam V, Abdullah N, Kuppusamy UR, Naidu M (2009) Effects of Cultivation Techniques and Processing on Antimicrobial and Antioxidan Activities of $H$. erinaceus. Food Technol Biotechnol 47: 47-55.

5. Kolotushkina EV, Moldavan MG, Voronin KY, Skibo GG (2003) The influence of Hericium erinaceus extract on myelination process in vitro. Fiziol $\mathrm{Zh}$ 49: 38-45.

6. Kawagishi H, Shimada A, Shirai R, Okamoto K, Ojima F, et al. (1994) Erinacines $A, B$ and $C$, strong stimulators of nerve growth factor (NGF)-synthesis, from the mycelia of Hericium erinaceum. Tetrahedron Letters 35: 1569-1572.

7. Kawagishi H, Shimada A, Shizuki K, Mori H (1996) Erinacine D, a stimulator of NGF-synthesis, from the mycelia of Hericium erinaceum. Heterocyclic Communications 2: 51-54.

8. Kawagishi H, Shimada A, Hosokawa S, Mori H, et al. (1996) Erinacines E, F, and G, stimulators of nerve growth factor (NGF)-synthesis, from the mycelia of Hericium erinaceum. Tetrahedron Letters 37: 7399-7402.

9. Kawagishi H, Masui A, Tokuyama S, Nakamura T (2006) Erinacines $\mathrm{J}$ and $\mathrm{K}$ from the mycelia of Hericium erinaceum. Tetrahedron 62: 8463-8466.

10. Lee EW, Shizuki K, Hosokawa S, Suzuki M, Suganuma H, et al. (2000) Two novel diterpenoids, erinacines $\mathrm{H}$ and I from the mycelia of Hericium erinaceum. Biosci Biotechnol Biochem 64: 2402-2405.

11. Kenmoku H, Sassa $T$, Kato $N$ (2000) Isolation of erinacine $P$, a new parental metabolite of cyathane-xylosides, from Hericium erinaceum and its biomimetic conversion into erinacines A and B. Tetrahedron Letters 41: 4389-4393.
12. Xing XW, Hawthorne WJ, Yi S, Simond DM, Dong Q, et al. (2009) Investigation of porcine endogenous retrovirus in the conservation population of Ningxiang pig. Transplant Proc 41: 4389-4393.

13. Kenmoku H, Shimai T, Toyomasu T, Kato N, Sassa T (2002) Erinacine Q, a new erinacine from Hericium erinaceum, and its biosynthetic route to erinacine $\mathrm{C}$ in the basidiomycete. Biosci Biotechnol Biochem 66: 571-575.

14. Riaz SS, Tomlinson DR (1996) Neurotrophic factors in peripheral neuropathies: pharmacological strategies. Prog Neurobiol 49: 125-143.

15. Yamada K, Nitta A, Hasegawa T, Fuji K, Hiramatsu M, et al. (1997) Orally active NGF synthesis stimulators: potential therapeutic agents in Alzheimer's disease. Behav Brain Res 83: 117-122.

16. Shimbo M, Kawagishi H, Yokogoshi H (2005) Erinacine A increases catecholamine and nerve growth factor content in the central nervous system of rats. Nutrition Research 25: 617-623.

17. Nagano M, Shimizu K, Kondo R, Hayashi C, Sato D, et al. (2010) Reduction of depression and anxiety by 4 weeks Hericium erinaceus intake. Biomed Res 31: 231-237.

18. Krzyczkowski W, Malinowska E, Herold F (2010) Erinacine A biosynthesis in submerged cultivation of Hericium erinaceum: Quantification and improved cultivation. Engineering in Life Sciences 10: 446-457.

19. Krzyczkowski W, Malinowska E, Suchocki P, Kleps J, Olejnik M, et al. (2009) Isolation and quantitative determination of ergosterol peroxide in various edible mushroom species. Food Chemistry 113: 351-355.

20. Maruyama H, Yamazaki K, Murofushi S, Konda C, Ikekawa T (1989) Antitumor activity of Sarcodon aspratus (Berk.) S. Ito and Ganoderma Iucidum (Fr.) Karst. J Pharmacobiodyn 12: 118-123.

21. Berovic M, Habijanic J, Boh B, Wraber B, Petravic-Tominac V (2012) Production of Lingzhi or Reishi medicinal mushroom, Ganoderma lucidum (W.Curt. :Fr.) P. Karst. (higher Basidiomycetes), biomass and polysaccharides by solid state cultivation. Int J Med Mushrooms 14: 513-520.

22. Klamer M, Baath E (2004) Estimation of conversion factors for fungal biomass determination in compost using ergosterol and PLFA 18:2w6,9. Soil Biology \& Biochemistry 36: 57-65.

23. Nyuland JE, Wallander $\mathrm{H}$ (1992) 5 Ergosterol analysis as a means of quantifying mycorrhizal biomass. Methods in Microbiology 24: 77-88. 